\title{
Salivary alpha amylase not chromogranin A reflects sympathetic activity: exercise responses in elite male wheelchair athletes with or without cervical spinal cord injury
}

\author{
Christof A. Leicht", Thomas A. W. Paulson, Victoria L. Goosey-Tolfrey and Nicolette C. Bishop
}

\begin{abstract}
Background: Salivary alpha amylase ( $\mathrm{sAA}$ ) and chromogranin A ( $\mathrm{s} \mathrm{CgA}$ ) have both been suggested as non-invasive markers for sympathetic nervous system (SNS) activity. A complete cervical spinal cord injury leading to tetraplegia is accompanied with sympathetic dysfunction; the aim of this study was to establish the exercise response of these markers in this in vivo model.

Methods: Twenty-six elite male wheelchair athletes (C6-C7 tetraplegia: $N=8, T 6-\mathrm{L} 1$ paraplegia: $N=10$ and non-spinal cord injured controls: $N=8$ ) performed treadmill exercise to exhaustion. Saliva and blood samples were taken pre, post and 30 min post exercise and analysed for sAA, SCgA and plasma adrenaline concentration, respectively.

Results: In all three subgroups, sAA and sCgA were elevated post exercise $(P<0.05)$. Whilst $\mathrm{s} C \mathrm{gA}$ was not different between subgroups, a group $\times$ time interaction for SAA explained the reduced post-exercise SAA activity in tetraplegia $\left(162 \pm 127\right.$ vs $313 \pm 99$ (paraplegia) and $328 \pm 131 \mathrm{U} \mathrm{mL}^{-1}$ (controls), $P=0.005$ ). The post-exercise increase in adrenaline was not apparent in tetraplegia $(P=0.74)$. A significant correlation was found between adrenaline and SAA $(r=0.60, P=0.01)$, but not between adrenaline and SCgA $(r=0.06, P=0.79)$.

Conclusions: The blunted post-exercise rise in SAA and adrenaline in tetraplegia implies that both reflect SNS activity to some degree. It is questionable whether SCgA should be used as a marker for SNS activity, both due to the exercise response which is not different between the subgroups and its non-significant relationship with adrenaline.
\end{abstract}

Keywords: Adrenaline, Catecholamines, Cortisol, Sympathetic dysfunction, Testosterone, Wheelchair athlete, Wheelchair propulsion

\section{Key points}

- This study shows a blunted alpha amylase but a normal chromogranin A response to maximal exercise in athletes with sympathetic dysfunction.

- This study would favour the use of alpha amylase over chromogranin A as a surrogate marker for sympathetic activity.

- However, in contrast to the adrenaline response, the alpha amylase response is not completely absent in

\footnotetext{
* Correspondence: c.a.leicht@lboro.ac.uk

School of Sport, Exercise, and Health Sciences, The Peter Harrison Centre for Disability Sport, The National Centre for Sport and Exercise Medicine, Loughborough University, Loughborough LE11 3TU, UK
}

cervical spinal cord injury, implying it is also regulated by mechanisms other than sympathetic activity.

\section{Background}

Salivary secretions provide a non-invasive alternative to blood-derived markers to quantify exercise stress in both clinical and sports performance settings. Whilst salivary catecholamines have been suggested to be poor markers of acute sympathetic nervous system (SNS) activity [1], the salivary proteins $\alpha$-amylase (sAA) and chromogranin A ( $\mathrm{sCgA})$ have been proposed to serve this purpose $[2,3]$. Indeed, sAA activity is responsive to exercise, particularly that of a high-intensity nature, supporting the relationship between sAA and SNS activity [4-7]. Further, adrenergic 
receptor blockade can inhibit the stress-induced secretion of sAA [8]. However, the correlations between sAA and catecholamines in the circulation are relatively small $[9,10]$. It has hence been questioned whether sAA truly reflects SNS activity as the influence of parasympathetic activity on sAA secretion may confound a stronger relationship $[9,11]$.

Chromogranin A is stored and co-released with adrenaline and noradrenaline from secretory vesicles within the adrenal medulla and post-ganglionic sympathetic axons [12]. It is also secreted from the submandibular gland following stimulation by noradrenaline and acetylcholine [13]. In response to acute exercise stress, sCgA shows a similar response to SAA $[5,14]$. However, the correlation to physiological responses during acute exercise has been shown to be stronger for SCgA than for sAA [2]. This suggests a differential regulation of the two proteins and that sCgA may provide a more accurate representation of SNS activity. Conversely, non-exercise stress in the form of noise [15] or delivering a lecture [16] has been shown to increase sAA, whereas $\mathrm{SCgA}$ remained unaffected, questioning to what extent $\mathrm{sCgA}$ is regulated by the SNS.

Cortisol and testosterone represent two further markers of exercise stress and recovery that can be measured in plasma as well as in saliva. In response to acute exercise, both markers increase in a time and intensity-dependent manner $[17,18]$. Cortisol and testosterone, expressed as a ratio, have also been put forward to describe the anaboliccatabolic balance. In this context, they may help in the diagnosis of the overtraining syndrome, even though it has been made clear that supporting markers are needed to define this condition [19].

The primary aim of this study was to investigate the impact of exercise to exhaustion on established plasma markers for SNS activity (adrenaline) and on proposed salivary markers for SNS activity (sAA and $\mathrm{sCgA}$ ) in a human in vivo model of SNS dysfunction. The model of spinal cord injury (SCI) was employed: A complete injury to the cervical region of the spinal cord results in a tetraplegia (TETRA) and the dysfunction of the SNS, as evidenced by reduced cardiac acceleration [20] or a reduced catecholamine concentration at rest or following exercise [21]. However, increases in sAA activity have been found previously in both cervical- and thoracic-level athletes with SCI and non-spinal injured controls following strenuous exercise, which further questions sAA as a true indicator of central sympathetic drive [22]. We hence hypothesise the exercise-induced changes in adrenaline to be more closely related to $\mathrm{SCgA}$ than to sAA.

The secondary aim of this study was to investigate the impact of exercise on plasma and salivary steroid hormones in the SCI model. Whilst cortisol secretion is governed by humoral mechanisms (the hypothalamuspituitary-adrenal or HPA axis), testosterone secretion is partly governed by neural pathways [23], explaining the high proportion of testosterone deficiency in the SCI population [24]. We hence hypothesise a normal plasma and salivary cortisol but a blunted testosterone response to exercise in TETRA.

\section{Methods \\ Participants}

The present data have not been previously published, but the samples were collected as part of a larger study [21]; as such, the participants studied and the exercise protocol employed are identical to this publication. Twentysix international-level male wheelchair athletes volunteered to participate and were grouped according to their disability, TETRA, $(N=8)$, paraplegia (PARA, $N=10)$ and disabilities unrelated to SCI (non-spinal injured (NON-SCI), $N=8$ ). All participants with SCI had a motor and sensory complete lesion in accordance with the American Spinal Injury Association (ASIA) impairment scale [25]. A summary of the participants' characteristics and their main peak responses to exercise are presented in Table 1. All procedures were approved by the Loughborough University Ethical Advisory Committee and were in accordance with the Declaration of Helsinki. Participants provided written informed consent prior to the experiments, and they were free from infectious symptoms and pressure sores.

\section{Experimental protocol}

Participants reported to the laboratory between 09:30 and 11:30 having been fasted for at least $2 \mathrm{~h}$. They were asked to refrain from strenuous physical activity and caffeine intake $24 \mathrm{~h}$ prior to exercise. On arrival, their body mass was obtained to the nearest $0.1 \mathrm{~kg}$ using doublebeam seated scales (Marsden MPWS-300, Rotherham, UK). All exercise tests were performed in the participants' competition court sports wheelchair on a motorised treadmill (HP Cosmos, Traunstein, Germany) as described previously [21]. First, a $\sim 30$-min warm-up at intensities covering a range from 40 to $80 \%$ peak oxygen uptake $\left(\dot{\mathrm{V}}_{2 \text { peak }}\right)$ was performed, followed by a 15 -min passive recovery. A graded exercise test to exhaustion (GXT) was then completed at a constant speed. The gradient at the start of the GXT was $1.0 \%$ for all subgroups, with subsequent increases of $0.3 \%$ every minute for PARA and NON-SCI and $0.1 \%$ every $40 \mathrm{~s}$ for TETRA to account for the functional differences between subgroups and ensure a minimum GXT duration of $\sim 8$ min. After the GXT, participants recovered actively at a low intensity $\left(1.2 \mathrm{~m} \mathrm{~s}^{-1}\right.$ at a $1.0 \%$ gradient) for $5 \mathrm{~min}$. Participants then performed a verification test, designed as a test to exhaustion at the same constant speed but 0.3 and $0.1 \%$ higher than the maximal gradient achieved during the GXT for NON-SCI/PARA and TETRA, respectively. The GXT and the verification test were terminated when participants 
Table 1 Participants' characteristics and peak exercise responses

\begin{tabular}{llll}
\hline & TETRA & PARA & $\mathrm{NON-SCl}$ \\
\hline Age (years) & $31 \pm 6$ & $30 \pm 8$ & $27 \pm 8$ \\
Body mass (kg) & $67.3 \pm 5.9^{\mathrm{a}}$ & $72.3 \pm 13.5$ & $84.8 \pm 10.7$ \\
Disability & $\mathrm{SCl} \mathrm{C6-7}$ & $\mathrm{SCl}$ T6-L1/spina bifida & Amputation/club foot \\
ASIA impairment scale & $\mathrm{A}$ & $\mathrm{A}$ & $\mathrm{N} / \mathrm{A}$ \\
Wheelchair sport & Rugby & Basketball & Basketball \\
Training $\left(\mathrm{h} \mathrm{week}^{-1}\right)$ & $13 \pm 2$ & $15 \pm 3$ & $16 \pm 2$ \\
$\dot{\mathrm{V}} \mathrm{O}_{2 \text { peak }}\left(\mathrm{L} \mathrm{min}^{-1}\right)$ & $1.44 \pm 0.32^{\mathrm{b}}$ & $2.85 \pm 0.87^{\mathrm{b}}$ & $3.75 \pm 0.33^{\mathrm{b}}$ \\
$\mathrm{HR}_{\text {peak }}\left(\mathrm{b} \mathrm{min}^{-1}\right)$ & $127 \pm 10^{\mathrm{b}}$ & $181 \pm 10$ & $183 \pm 8$ \\
BLa $_{\text {peak }}\left(\mathrm{mmol} \mathrm{L}^{-1}\right)$ & $5.39 \pm 0.96^{\mathrm{b}}$ & $7.69 \pm 1.87$ & $8.29 \pm 1.64$ \\
RPE $_{\text {peak }}$ & $20(19-20)$ & $19(19-20)$ & $19(19-20)$
\end{tabular}

Data are mean \pm SD or median (interquartile range); $P<0.05$

TETRA tetraplegia, PARA paraplegia, NON-SCI non-spinal injured, ASIA American Spinal Injury Association, $H R$ heart rate, BLa blood lactate concentration, RPE rating of perceived exertion, $\mathrm{SCl}$ spinal cord injury

asignificantly different from NON-SCI

${ }^{\mathrm{b}}$ Significantly different from other subgroups

were unable to maintain the speed of the treadmill. Verbal encouragement was given throughout the test, and participants were allowed to consume water ad libitum during the procedure.

\section{Data collection}

Expired air was collected for at least the final three consecutive minutes of the GXT and for 2 min during the verification test and analysed using the Douglas bag technique, using a gas analyser (Series 1400; Servomex Ltd, Sussex, UK) and a dry gas meter (Harvard Apparatus, Kent, UK). Blood lactate concentration (BLa) was determined using a calibrated lactate analyser (YSI 1500 SPORT, YSI Incorporated, Yellow Springs, OH, USA) from a capillary blood sample obtained immediately after the GXT and immediately after the verification test. At the same time points, participants were asked to indicate an overall rating of perceived exertion (RPE) using the 15-point Borg scale according to previous instructions. Heart rate was continuously recorded at 5-s intervals (Polar PE 4000, Polar, Kempele, Finland). The higher of the two $\dot{\mathrm{VO}}_{2 \text { peak }}, \mathrm{HR}_{\text {peak }}$ and $\mathrm{BLa}_{\text {peak }}$ values obtained in the GXT and the verification test was taken as peak value. All participants had prior experience of the physiological testing procedure and were therefore familiar with the protocol.

Blood and saliva samples were collected before (pre), immediately after the verification test (post) and $30 \mathrm{~min}$ after exercise (post30). A 4.9-mL blood sample was drawn from an antecubital vein into a $K_{3}$ EDTA vacutainer. Timed, unstimulated saliva samples were collected as described previously [22]; the participants' head slightly tilted forward with minimal orofacial movement during collection after rinsing their mouth out with water immediately before collection. Participants were allowed to consume water ad libitum apart from 6 min before each collection. Saliva flow rate was calculated by dividing the obtained saliva volume by the collection time.

\section{Plasma and saliva analysis}

Blood samples were refrigerated until the final sample from each participant was collected and then centrifuged at $1500 \mathrm{~g}$ for $10 \mathrm{~min}\left(\right.$ at $4{ }^{\circ} \mathrm{C}$ ). Whole saliva samples were immediately centrifuged at 13,000 rpm for 2 min following collection. The separated plasma was then immediately stored at $-80{ }^{\circ} \mathrm{C}$ and saliva at $-20{ }^{\circ} \mathrm{C}$. Plasma concentrations of adrenaline, cortisol (pCort) and free testosterone (pTest) were determined using quantitative sandwichtype enzyme-linked immunosorbant assay (ELISA) kits (adrenaline: IBL international, Hamburg, Germany; $p$ Cort and pTest: DRG instruments, Marburg, Germany), according to the manufacturers' instructions. All samples were analysed in duplicate. Saliva concentrations of cortisol (sCort), testosterone (sTest) and sCgA were also determined using ELISA (sCort and sTest: Salimetrics, Newmarket, UK; $s C g A$ : Demeditic Diagnostics GmbH, Kiel, Germany) and sAA with an enzymatic activity assay as described previously [22]. All samples from one participant were analysed on the same ELISA plate; the withinassay coefficient of variation for the analyses performed was as follows: adrenaline $2.7 \%$, pCort $1.3 \%$, pTest $4.5 \%$, sCort $2.8 \%$, sTest $3.7 \%$, sCgA $5.2 \%$ and sAA $4.9 \%$.

\section{Statistical analysis}

Data were analysed using IBM SPSS for Windows version 19 (SPSS inc, Chicago, IL). Exercise responses were analysed using a two-way mixed measures ANOVA with time as within- and group as between-measures variable for normally distributed variables, and significant main effects were assessed using Sidak post hoc tests. Adrenaline and 
sTest data were analysed using non-parametric Friedman tests for each group separately; adrenaline differences were assessed using Mann-Whitney $U$ tests between TETRA and the other two subgroups (with intact sympathetic function) combined. Pearson-product moment correlations for normally distributed data and Spearman's rho for non-normally distributed data were used to determine the relationship between plasma and salivary concentrations of cortisol and testosterone, as well as between adrenaline and the salivary proteins $\mathrm{sAA}$ and $\mathrm{SCgA}$. Significance was set at $P \leq 0.05$, and Bonferroni adjustments were performed when performing multiple comparisons. Data are presented as mean \pm standard deviation.

\section{Results}

A significant effect of time was found for plasma adrenaline concentration for both PARA and NON-SCI with a 2.2-fold increase from pre $(P<0.05)$ but not for TETRA $(P=0.74$, Fig. 1 , Table 2). Furthermore, the plasma adrenaline concentration was significantly lower in TETRA when compared with the other subgroups with intact sympathetic function $(P<0.001)$. The concentrations of sAA were highest at post exercise in all subgroups (2.3fold increase from pre, $P<0.03$ ), a main effect of group was found in sAA with lower values in TETRA than in PARA $(P=0.02)$ and a group $\times$ time interaction indicated a blunted response in TETRA $(P=0.005)$. The same pattern was found for the sAA secretion rate, with the exception that a main effect of group indicated lowest values for TETRA when compared with both other subgroups $(P<0.05)$. Post-exercise concentrations of sCgA were significantly elevated in all subgroups (threefold from pre, $P<0.001$ ) with no difference between subgroups $(P=0.69)$. TETRA had a lower saliva flow rate than NON-SCI $(P=0.02)$, the saliva flow rate of PARA was not different from the other subgroups $(P>0.33)$. Saliva flow rate did not change over time $(P=0.77)$.

Correlations between plasma adrenaline concentration and salivary parameter post exercise were significant for sAA $(r=0.60, P=0.01)$, but not for $\mathrm{sCgA}(r=0.06, P=0.79$, Fig. 2). The correlation between sAA and sCgA was also not significant $(r=0.25, P=0.23)$.

Plasma and salivary steroid concentrations are shown in Fig. 3 and in Table 2. Significant effects of time $(P<0.001)$ demonstrate an increase in both plasma and salivary cortisol concentrations following exercise, with the highest concentrations found at post and post30 for plasma (both 1.4-fold elevated from pre), whilst salivary concentrations were only elevated at post30 (1.9-fold from pre, $P<0.01$ ). No significant group $(P>0.89)$ or interaction $(P>0.68)$ effects were found for plasma and salivary cortisol concentrations. A main effect of time was found for pTest $(P=0.02)$ which only showed a trend for post concentrations to be elevated from the other time points (1.3-fold

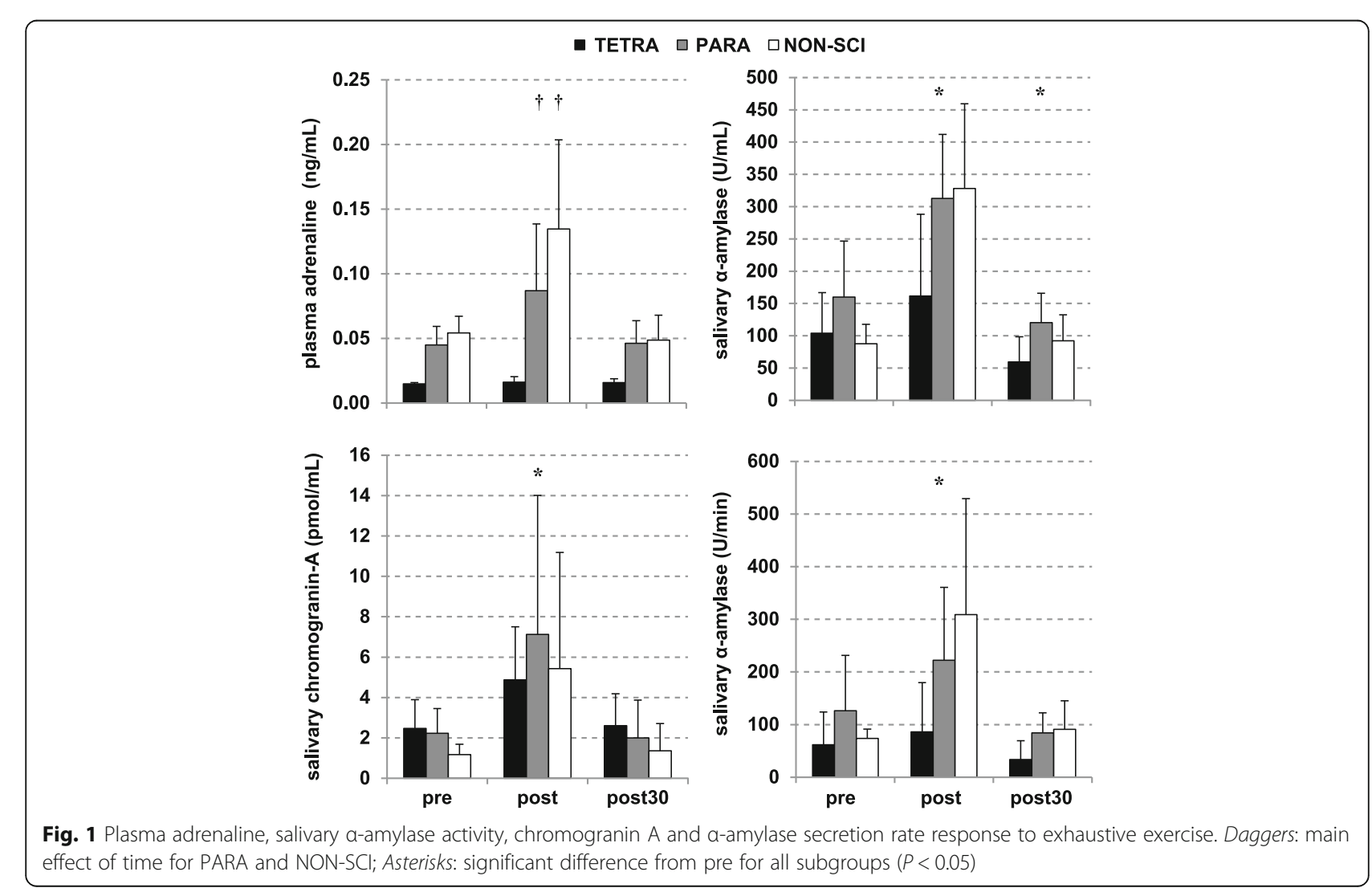


Table 2 Plasma and salivary responses to exhaustive exercise

\begin{tabular}{|c|c|c|c|c|}
\hline Parameter (unit) & Time & TETRA & PARA & $\mathrm{NON}-\mathrm{SCl}$ \\
\hline \multirow[t]{3}{*}{ Plasma adrenaline concentration ( $\mathrm{ng} / \mathrm{mL})$} & Pre & $0.02 \pm 0.00$ & $0.05 \pm 0.01$ & $0.05 \pm 0.01$ \\
\hline & Post & $0.02 \pm 0.00$ & $0.09 \pm 0.05^{\mathrm{a}}$ & $0.14 \pm 0.07^{\mathrm{a}}$ \\
\hline & Post30 & $0.02 \pm 0.00$ & $0.05 \pm 0.02$ & $0.05 \pm 0.02$ \\
\hline \multirow[t]{3}{*}{ Salivary chromogranin A concentration (pmol/mL) } & Pre & $2.47 \pm 1.23$ & $2.23 \pm 1.22$ & $1.18 \pm 0.51$ \\
\hline & Post & $4.88 \pm 2.63^{\mathrm{a}}$ & $7.12 \pm 6.88^{\mathrm{a}}$ & $5.44 \pm 5.75^{\mathrm{a}}$ \\
\hline & Post30 & $2.61 \pm 1.57$ & $2.00 \pm 1.87$ & $1.37 \pm 1.35$ \\
\hline \multirow[t]{3}{*}{ Salivary a-amylase activity (U/mL) } & Pre & $104 \pm 63$ & $160 \pm 87$ & $88 \pm 30$ \\
\hline & Post & $162 \pm 127^{a}$ & $313 \pm 99^{a}$ & $328 \pm 131^{a}$ \\
\hline & Post30 & $59 \pm 39^{a}$ & $120 \pm 46^{\mathrm{a}}$ & $92 \pm 40^{\mathrm{a}}$ \\
\hline \multirow[t]{3}{*}{ Salivary a-amylase secretion rate (U/min) } & Pre & $62 \pm 62$ & $126 \pm 105$ & $74 \pm 18$ \\
\hline & Post & $86 \pm 93^{\mathrm{a}}$ & $222 \pm 138^{\mathrm{a}}$ & $309 \pm 220^{\mathrm{a}}$ \\
\hline & Post30 & $34 \pm 36$ & $84 \pm 38$ & $91 \pm 54$ \\
\hline \multirow[t]{3}{*}{ Plasma cortisol concentration (ng/mL) } & Pre & $128 \pm 13$ & $113 \pm 32$ & $125 \pm 33$ \\
\hline & Post & $178 \pm 36^{\mathrm{a}}$ & $184 \pm 72^{a}$ & $169 \pm 33^{\mathrm{a}}$ \\
\hline & Post30 & $173 \pm 64^{a}$ & $171 \pm 58^{\mathrm{a}}$ & $160 \pm 47^{a}$ \\
\hline \multirow[t]{3}{*}{ Salivary cortisol concentration (ng/mL) } & Pre & $5.48 \pm 0.99$ & $5.60 \pm 1.75$ & $5.46 \pm 3.71$ \\
\hline & Post & $5.87 \pm 1.66$ & $6.05 \pm 2.65$ & $6.47 \pm 3.71$ \\
\hline & Post30 & $9.88 \pm 6.24^{\mathrm{ab}}$ & $11.69 \pm 7.48^{\mathrm{ab}}$ & $8.99 \pm 4.23^{\mathrm{ab}}$ \\
\hline \multirow[t]{3}{*}{ Plasma testosterone concentration (ng/mL) } & Pre & $12.0 \pm 4.6$ & $10.9 \pm 7.2$ & $12.6 \pm 5.4$ \\
\hline & Post & $15.8 \pm 6.0$ & $16.1 \pm 17.4$ & $15.5 \pm 6.1$ \\
\hline & Post30 & $15.4 \pm 6.8$ & $12.7 \pm 12.7$ & $11.6 \pm 3.4$ \\
\hline \multirow[t]{3}{*}{ Salivary testosterone concentration $(\mathrm{pg} / \mathrm{mL})$} & Pre & $128 \pm 21$ & $126 \pm 32$ & $107 \pm 50$ \\
\hline & Post & $138 \pm 15$ & $131 \pm 39$ & $150 \pm 36$ \\
\hline & Post30 & $141 \pm 23$ & $122 \pm 24$ & $131 \pm 33$ \\
\hline \multirow[t]{3}{*}{ Saliva flow rate (mL/min) } & Pre & $0.52 \pm 0.22$ & $0.78 \pm 0.54$ & $0.95 \pm 0.43$ \\
\hline & Post & $0.45 \pm 0.18$ & $0.72 \pm 0.40$ & $0.95 \pm 0.45$ \\
\hline & Post30 & $0.51 \pm 0.22$ & $0.72 \pm 0.27$ & $0.99 \pm 0.33$ \\
\hline
\end{tabular}

Data are mean \pm SD; $P<0.05$

${ }^{\mathrm{a}}$ Significantly different from pre

${ }^{\mathrm{b}}$ Significantly different from post

from pre, $P=0.06$ ), whereas no group or time effects were found for sTest $(P>0.05)$.

Significant correlations were found between plasma and salivary concentrations of cortisol both at pre $(r=0.60$, $P=0.003)$ and post30 $(r=0.68, P<0.001)$, whereas a trend was evident at post $(r=0.43, P=0.09)$. In contrast, no significant correlation was found between corresponding plasma and salivary testosterone concentrations (pre: $r=0.08$; post: $r=0.34$; post $30: r=0.21 ; P>0.05)$.

\section{Discussion}

The main findings of the present study were that (1) both sAA and sCgA concentrations increased following exhaustive exercise in all spinal injury level subgroups; (2) the post-exercise sAA response was blunted in TETRA; (3) post-exercise plasma adrenaline concentrations were significantly correlated with post-exercise sAA concentrations, but not with sCgA concentrations; (4) in contrast to the other subgroups, the plasma adrenaline concentrations in TETRA did not alter in response to exercise; and (5) salivary cortisol but not testosterone reflected plasma activity, the responses not being different between subgroups.

\section{Are $\mathrm{sAA}$ and $\mathrm{s} \mathrm{CgA}$ appropriate salivary markers for sympathetic activity?}

The primary aim of this study was to investigate the impact of strenuous exercise on established plasma (adrenaline) and salivary (sAA and $\mathrm{sCgA}$ ) markers for SNS activity in a human in vivo model of SNS dysfunction. The present results support the finding that sAA is partly governed by SNS activity [11], as a blunted response was observed in TETRA when compared with the other subgroups. The correlation between adrenaline and sAA 


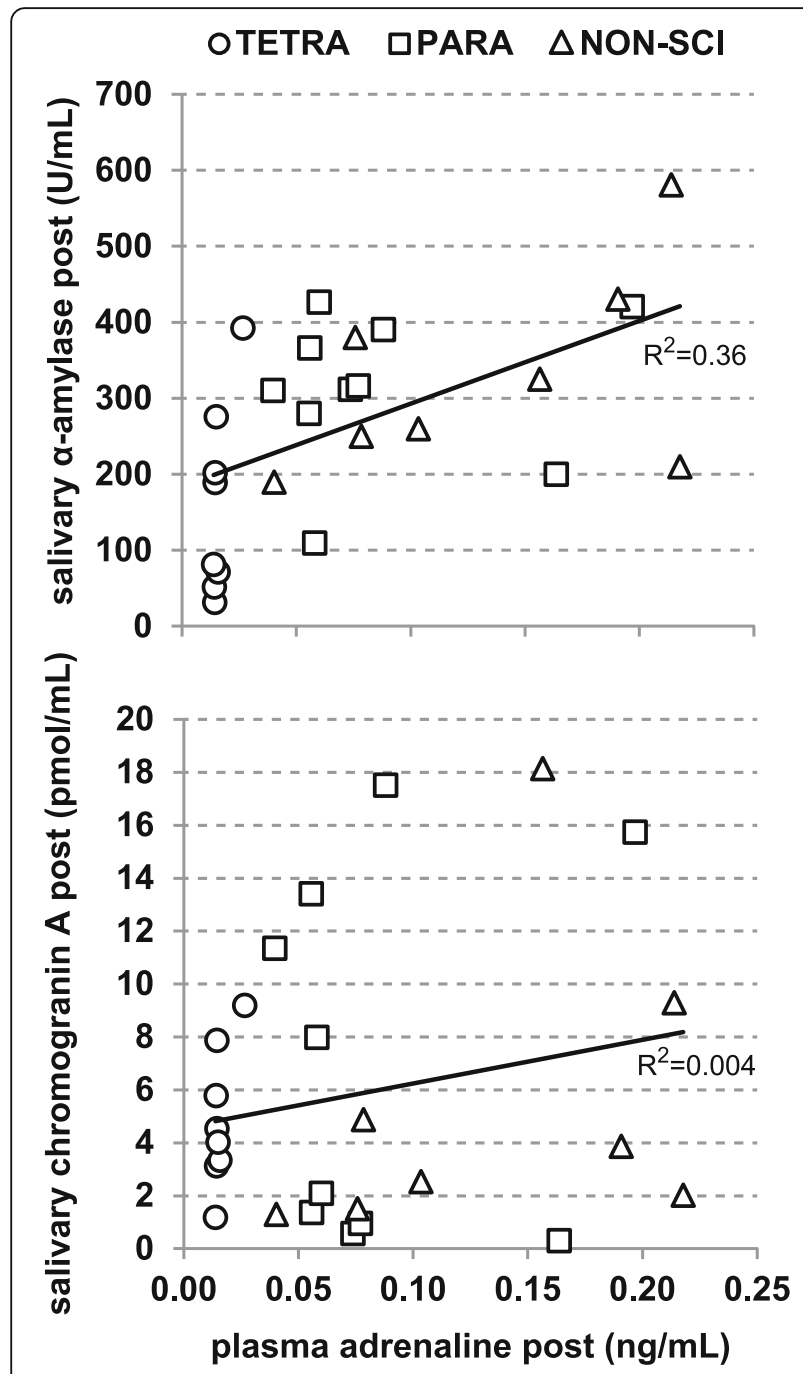

Fig. 2 Suggested salivary markers for SNS activity and their relationship with plasma adrenaline after exhaustive exercise. $R^{2}$ coefficient of determination

further imply that sAA could be used as a surrogate marker for adrenaline. However, the variance explained was only $36 \%\left(R^{2}\right)$, leaving a considerable amount of unexplained random variation, which is of a similar magnitude as found previously [9]. It is likely that some of this random variation is explained by the contribution of the parasympathetic nervous system [11], which contributes to sAA, but not to adrenaline release. Therefore, whilst plasma catecholamine concentrations represent a gold standard with respect to SNS activity, sAA activity may be used to help indicating SNS dysfunction in an exercise context. These results are in contrast to earlier findings, where no significant differences between the same subgroups as investigated in the current study have been found with respect to SAA activity following strenuous interval exercise [22]. However, closer inspection of these previous data reveals a $22 \%$ lower sAA activity in TETRA post exercise, even though the difference to the subgroups with intact SNS was insignificant. It should further be noted that the exercise performed during this previous study was not performed to exhaustion as in the current study. This could further explain the previous non-significant difference, as the subgroups with intact SNS activity were likely not to initiate the full potential of their sAA response. Indeed, exercise of an incremental nature with continuous data sampling shows a continuous increase in sAA and $\mathrm{sCgA}$ concentration with increasing exercise intensity which led to the suggestion for their use as markers for exercise intensity [14].

Whilst sAA responds to stressors other than exercise, such as emotional stressors [9], it is worth noting that Bocanegra et al. [14] suggest sAA and sCgA as markers for exercise intensity, not sympathetic activity in exercising contexts. This is an aspect worth developing: correlation (between salivary markers and exercise intensity) does not necessarily imply causation (of their regulation by the SNS), as demonstrated by comparing the present findings with the data presented by Bocanegra et al. [14]. Despite the strong relationships between $\mathrm{sAA}, \mathrm{sCgA}$ and exercise intensity [14], factors other than SNS activity, such as parasympathetic activity, reflex activity or, in the case of SCI, receptor hypersensitivity [26], may also contribute to the changes observed in these salivary markers in response to exercise. Therefore, SNS activity cannot be suggested as their main modulating component. This thought may be further developed to question the use of $\mathrm{sCgA}$ as a marker of SNS activity as previously suggested [2], as the present data do not support sCgA as a marker for SNS activity for two reasons: first, the exercise response is not different between the wheelchair athlete subgroups, and second, the relationship with adrenaline is insignificant.

The steroid hormone and saliva flow rate response in $\mathrm{SCl}$ The cortisol exercise response was not different between subgroups. This is consistent with the humoral regulation of cortisol release, which is independent of SNS function. In contrast, testosterone is secreted partly through the action of neuronal mechanisms [23], which again lends itself to be studied with the SCI model. However, no group or interaction effects were found for the testosterone responses, implying that testosterone regulation is only minimally, if at all, dependent on neural activity in the context of an exercise intervention.

The present cortisol data corroborate earlier results: A positive association between salivary and circulating plasma/ serum cortisol concentrations has been confirmed both at rest [27-29] and following exercise [30-33]. Further, we have shown that exercise induces a rise in plasma cortisol concentration immediately following exercise, a response which can be observed with a time lag in the 

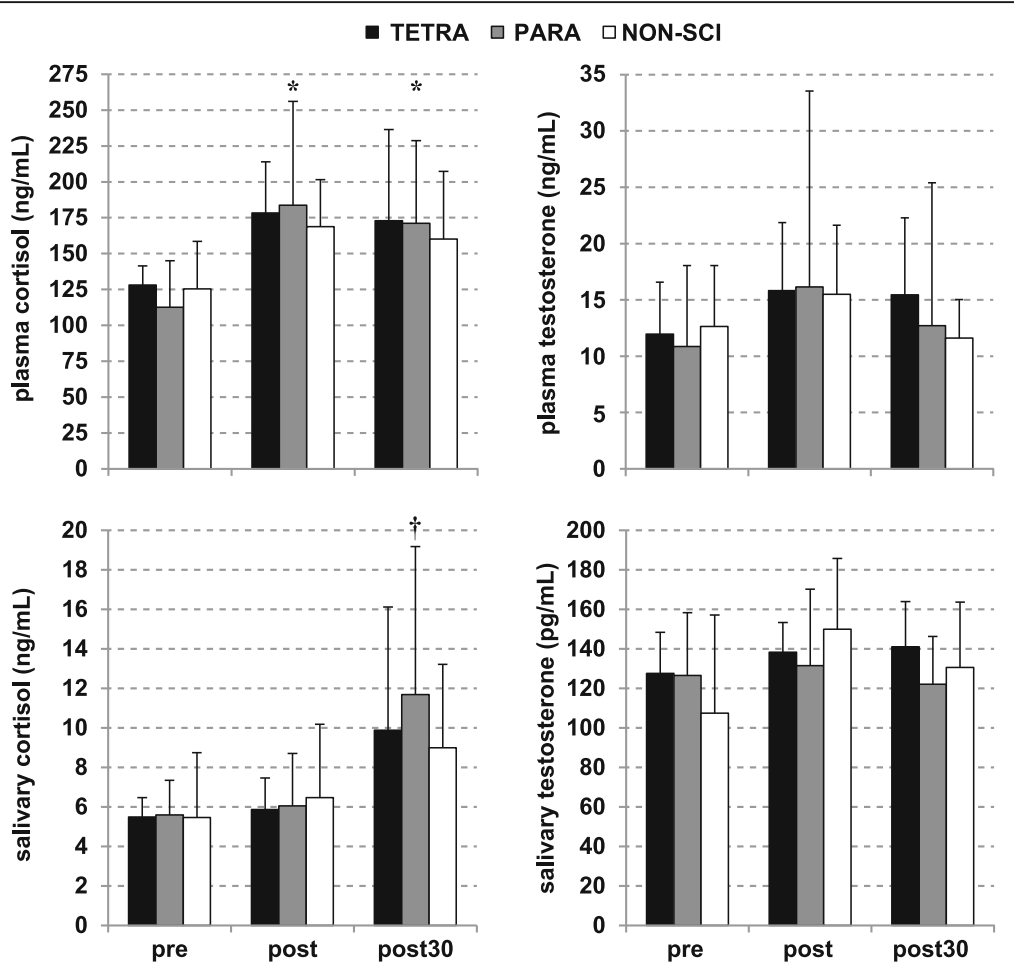

Fig. 3 Cortisol and testosterone response to exhaustive exercise. Asterisks: significant difference from pre for all subgroups; Dagger: significant difference from pre and post for all subgroups $(P<0.05)$

salivary concentrations. Similar effects have been found previously in direct comparisons of plasma and salivary cortisol concentrations [34], and exercise studies report that the sCort peak trails the pCort peak, which is usually found soon after cessation of exercise [33]. As sCort originates from the circulation, this lag is likely due to the process of cortisol diffusion and ultrafiltration through acinar cells [35], and the lag between pCort and sCort peak values has been reported to be in the area of $\sim 10-20 \mathrm{~min}$ [33]. This is consistent with our findings-the weakest correlation between pCort and sCort was found post exercise, where the sCort concentration had not reached its maximum yet. It is therefore likely that the correlation between these two markers could be improved when comparing post-exercise pCort values with sCort sampled with a delay of $10-20 \mathrm{~min}$. Including a sampling lag for sCort may also be relevant whenever the focus lies on determining the true maximum cortisol value, for example in the context of quantifying acute exercise stress [33] or in the context of over-reaching [36].

A modest but significant increase was observed in pTest, but not in sTest for all subgroups. The increases in pTest are consistent with previous reports [33], therefore suggesting that pTest may be used in SCI subgroups for the same diagnostic purposes as in the able-bodied population. The poor relationships between salivary and plasma testosterone also support previous research that questions the diagnostic value of salivary testosterone [35]; even though significant relationships have been shown between salivary and plasma/serum concentrations [27, 32], this was not replicated by others [33]. From a clinical perspective, the present study does not provide any evidence for testosterone deficiency in TETRA as previously reported [24]. However, previous research shows that exercise training increases resting testosterone levels in chronic SCI [37]; the highly trained nature of the investigated participant group is hence a likely explanation of this finding.

Finally, the reductions in saliva flow rate in TETRA underline the physiological difference of this subgroupsaliva flow rate can be increased by adrenoreceptor agonists but decreased by adrenoreceptor blocking drugs; furthermore, it is affected by neural stimulation [38]. The chronically lower adrenaline concentrations and interruption of neural pathways to the salivary glands in TETRA are a likely reason for these observed reductions in saliva flow rate.

\section{Future directions}

In addition to adrenaline, a number of studies report relationships between noradrenaline and suggested markers for SNS activity $[3,13]$. Whilst noradrenaline was not measured in the present study, it is highly likely that the noradrenaline response was similarly blunted as the 
adrenaline response, as shown earlier for cervical SCI $[39,40]$. Despite this, we suggest assessing the noradrenaline response in follow-up studies, which would allow a distinction between secretion mechanisms governed mainly by the adrenal glands (adrenaline) or secretion by sympathetic nerve endings (noradrenaline).

\section{Conclusions}

The blunted post-exercise rise in sAA and adrenaline in TETRA implies that both reflect SNS activity to some degree. Even though the sAA response to exercise is not absent, as is the case for adrenaline, sAA appears to reflect some of the SNS dysfunction found in TETRA. Therefore, rather than describing sAA as a marker of SNS activity in its own right, it would be more accurate to refer to it as a marker which partly reflects SNS activity but is also regulated by other mechanisms. Despite this limitation, we suggest that to date, sAA is the best surrogate salivary marker for SNS activity, which is relevant in the absence of available blood tests and if restricted to salivary analyses. This is in contrast to earlier findings which have proposed that $\mathrm{sCgA}$ may provide a more accurate representation of SNS activity than sAA [2] but supports the findings from non-exercise stress interventions that failed to observe increases in $\mathrm{sCgA}$ in the presence of sAA elevations $[15,16]$.

On a final note, plasma and salivary cortisol and testosterone responses to exercise did not differ between subgroups, implying a minimal involvement of sympathetic innervation in the acute exercise response.

\section{Abbreviations \\ BLa: Blood lactate concentration; GXT: Graded exercise test to exhaustion; HPA: Hypothalamus-pituitary-adrenal; HR: Heart rate; NON-SCl: Non-spinal injured; PARA: Paraplegia; pCort: Plasma cortisol; pTest: Plasma testosterone; RPE: Rating of perceived exertion; sAA: Salivary alpha amylase; sCgA: Salivary chromogranin A; SCl: Spinal cord injury; sCort: Salivary cortisol; \\ SNS: Sympathetic nervous system; sTest: Salivary testosterone; TETRA: Tetraplegia}

\section{Acknowledgements}

We thank the volunteers for their participation in this study.

\section{Funding}

We thank the Peter Harrison Centre for Disability Sport for financial support Nicolette Bishop is supported by the National Institute for Health Research (NIHR) Diet, Lifestyle and Physical Activity Biomedical Research Unit based at the University Hospitals of Leicester and Loughborough University. The views expressed are those of the authors and not necessarily those of the NHS, the NIHR or the Department of Health.

\section{Authors' contributions}

All authors were involved in the conception and planning of the study. CL was involved in the data analysis and manuscript preparation. TP was involved in the data collection and manuscript review. VT and NB were involved in the manuscript review. All authors read and approve the final manuscript.

\section{Competing interests}

Christof Leicht, Thomas Paulson, Victoria Goosey-Tolfrey and Nicolette Bishop declare that they have no conflict of interest.
Consent for publication

Not applicable

\section{Ethics approval and consent to participate}

This study was approved by the Loughborough University Ethical Advisory Committee, and all participants gave written informed consent prior to the experiments.

Received: 30 June 2016 Accepted: 8 December 2016

Published online: 04 January 2017

\section{References}

1. Kennedy B, Dillon E, Mills PJ, Ziegler MG. Catecholamines in human saliva, Life Sci. 2001;69(1):87-99.

2. Gallina S, Di Mauro M, D'Amico MA, D'Angelo E, Sablone A, Di Fonso A, et al. Salivary chromogranin A, but not alpha-amylase, correlates with cardiovascular parameters during high intensity exercise. Clin Endocrinol (Oxf). 2011;75(6):747-52.

3. Chatterton Jr RT, Vogelsong KM, Lu YC, Ellman AB, Hudgens GA. Salivary alpha-amylase as a measure of endogenous adrenergic activity. Clin Physiol. 1996;16(4):433-48.

4. $\mathrm{Li} \mathrm{TL}$, Gleeson $\mathrm{M}$. The effect of single and repeated bouts of prolonged cycling and circadian variation on saliva flow rate, immunoglobulin $\mathrm{A}$ and alpha-amylase responses. J Sports Sci. 2004;22(11-12):1015-24.

5. Allgrove JE, Gomes E, Hough J, Gleeson M. Effects of exercise intensity on salivary antimicrobial proteins and markers of stress in active men. J Sports Sci. 2008;26(6):653-61

6. Walsh NP, Blannin AK, Clark AM, Cook L, Robson PJ, Gleeson M. The effects of high-intensity intermittent exercise on saliva IgA, total protein and alpha-amylase. J Sports Sci. 1999;17(2):129-34.

7. Bishop NC, Walker GJ, Scanlon GA, Richards S, Rogers E. Salivary IgA responses to prolonged intensive exercise following caffeine ingestion. Med Sci Sports Exerc. 2006;38(3):513-9.

8. van Stegeren A, Rohleder N, Everaerd W, Wolf OT. Salivary alpha amylase as marker for adrenergic activity during stress: effect of betablockade. Psychoneuroendocrinology. 2006;31(1):137-41.

9. Nater UM, Rohleder N. Salivary alpha-amylase as a non-invasive biomarker for the sympathetic nervous system: current state of research. Psychoneuroendocrinology. 2009;34(4):486-96.

10. Nater UM, La Marca R, Florin L, Moses A, Langhans W, Koller MM, et al. Stress-induced changes in human salivary alpha-amylase activity-associations with adrenergic activity. Psychoneuroendocrinology. 2006;31(1):49-58.

11. Bosch JA, Veerman EC, de Geus EJ, Proctor GB. Alpha-amylase as a reliable and convenient measure of sympathetic activity: don't start salivating just yet! Psychoneuroendocrinology. 2011;36(4):449-53.

12. Zhang K, Rao F, Wen G, Salem RM, Vaingankar S, Mahata M, et al. Catecholamine storage vesicles and the metabolic syndrome: the role of the chromogranin A fragment pancreastatin. Diabetes Obes Metab. 2006;8(6):621-33.

13. Kanno T, Asada N, Yanase H, Iwanaga T, Ozaki T, Nishikawa Y, et al. Salivary secretion of highly concentrated chromogranin $\mathrm{A}$ in response to noradrenaline and acetylcholine in isolated and perfused rat submandibular glands. Exp Physiol. 1999;84(6):1073-83.

14. Bocanegra OL, Diaz MM, Teixeira RR, Soares SS, Espindola FS. Determination of the lactate threshold by means of salivary biomarkers: chromogranin A as novel marker of exercise intensity. Eur J Appl Physiol. 2012;112(9):3195-203.

15. Wagner J, Cik M, Marth E, Santner BI, Gallasch E, Lackner A, et al. Feasibility of testing three salivary stress biomarkers in relation to naturalistic traffic noise exposure. Int J Hyg Environ Health. 2010;213(2):153-5.

16. Filaire E, Dreux B, Massart A, Nourrit B, Rama LM, Teixeira A. Salivary alphaamylase, cortisol and chromogranin $A$ responses to a lecture: impact of sex Eur J Appl Physiol. 2009;106(1):71-7.

17. Jacks DE, Sowash J, Anning J, McGloughlin T, Andres F. Effect of exercise at three exercise intensities on salivary cortisol. J Strength Cond Res. 2002;16(2):286-9.

18. Crewther B, Cronin J, Keogh J, Cook C. The salivary testosterone and cortisol response to three loading schemes. J Strength Cond Res. 2008;22(1):250-5.

19. Urhausen A, Kindermann W. Diagnosis of overtraining: what tools do we have? Sports Med. 2002;32(2):95-102.

20. Krassioukov A. Autonomic function following cervical spinal cord injury Respir Physiol Neurobiol. 2009;169(2):157-64. 
21. Paulson TAW, Goosey-Tolfrey VL, Lenton JP, Leicht CA, Bishop NC. Spinal cord injury level and the circulating cytokine response to strenuous exercise. Med Sci Sports Exerc. 2013;45(9):1649-55.

22. Leicht CA, Bishop NC, Goosey-Tolfrey VL. Mucosal immune responses to treadmill exercise in elite wheelchair athletes. Med Sci Sports Exerc. 2011:43(8):1414-21.

23. Lee $\mathrm{S}$, Miselis R, Rivier C. Anatomical and functional evidence for a neural hypothalamic-testicular pathway that is independent of the pituitary. Endocrinology. 2002;143(11):4447-54.

24. Durga A, Sepahpanah F, Regozzi M, Hastings J, Crane DA. Prevalence of testosterone deficiency after spinal cord injury. PM R. 2011;3(10):929-32.

25. Roberts TT, Leonard GR, Cepela DJ. Classifications in brief: American Spinal Injury Association (ASIA) impairment scale. Clin Orthop Relat Res. 2016. doi:10.1007/s11999-016-5133-4.

26. Leicht CA, Goosey-Tolfrey VL, Bishop NC. Spinal cord injury: known and possible influences on the immune response to exercise. Exerc Immunol Rev. 2013;19:146-65.

27. Lippi G, Dipalo M, Buonocore R, Gnocchi C, Aloe R, Delsignore R. Analytical evaluation of free testosterone and cortisol immunoassays in saliva as a reliable alternative to serum in sports medicine. J Clin Lab Anal. 2016. doi:10.1002/jcla.21929.

28. Neary JP, Malbon L, McKenzie DC. Relationship between serum, saliva and urinary cortisol and its implication during recovery from training. J Sci Med Sport. 2002;5(2):108-14.

29. Rantonen PJ, Penttila I, Meurman JH, Savolainen K, Narvanen S, Helenius T. Growth hormone and cortisol in serum and saliva. Acta Odontol Scand. 2000;58(6):299-303.

30. Port K. Serum and saliva cortisol responses and blood lactate accumulation during incremental exercise testing. Int J Sports Med. 1991;12(5):490-4.

31. Thomasson R, Baillot A, Jollin L, Lecoq AM, Amiot V, Lasne F, et al. Correlation between plasma and saliva adrenocortical hormones in response to submaximal exercise. J Physiol Sci. 2010;60(6):435-9.

32. Cadore E, Lhullier F, Brentano M, Silva E, Ambrosini M, Spinelli R, et al. Correlations between serum and salivary hormonal concentrations in response to resistance exercise. J Sports Sci. 2008;26(10):1067-72.

33. Hough JP, Papacosta E, Wraith E, Gleeson M. Plasma and salivary steroid hormone responses of men to high-intensity cycling and resistance exercise. J Strength Cond Res. 2011;25(1):23-31.

34. Hernandez CE, Thierfelder T, Svennersten-Sjaunja K, Berg C, Orihuela A, Lidfors $L$. Time lag between peak concentrations of plasma and salivary cortisol following a stressful procedure in dairy cattle. Acta Vet Scand. 2014;56:61.

35. Wood P. Salivary steroid assays_-research or routine? Ann Clin Biochem. 2009;46(Pt 3):183-96.

36. Hough J, Corney R, Kouris A, Gleeson M. Salivary cortisol and testosterone responses to high-intensity cycling before and after an 11-day intensified training period. J Sports Sci. 2013;31(14):1614-23.

37. Rosety-Rodriguez M, Rosety I, Fornieles G, Rosety JM, Elosegui S, Rosety MA, et al. A short-term arm-crank exercise program improved testosterone deficiency in adults with chronic spinal cord injury. Int Braz J Urol. 2014;40(3):367-72.

38. Proctor GB, Garrett JR, Carpenter GH, Ebersole LE. Salivary secretion of immunoglobulin A by submandibular glands in response to autonomimetic infusions in anaesthetised rats. J Neuroimmunol. 2003;136(1-2):17-24.

39. Schmid A, Schmidt-Trucksass A, Huonker M, Konig D, Eisenbarth I, Sauerwein H, et al. Catecholamines response of high performance wheelchair athletes at rest and during exercise with autonomic dysreflexia. Int I Sports Med. 2001;22(1):2-7.

40. Schmid A, Huonker M, Barturen JM, Stahl F, Schmidt-Trucksass A, Konig D, et al. Catecholamines, heart rate, and oxygen uptake during exercise in persons with spinal cord injury. J Appl Physiol. 1998;85(2):635-41.

\section{Submit your manuscript to a SpringerOpen ${ }^{\circ}$ journal and benefit from:}

- Convenient online submission

- Rigorous peer review

- Immediate publication on acceptance

- Open access: articles freely available online

- High visibility within the field

- Retaining the copyright to your article

Submit your next manuscript at $\gg$ springeropen.com 